\title{
Room to breathe
}

Pierre Sonveaux, Olivier Feron, Mark Dewhirst and colleagues have identified a symbiosis involving lactate exchange between tumour cells that enlist different metabolic pathways to generate ATP.

The ability of hypoxic tumour cells to uncouple glycolysis from respiration as a means for continued generation of ATP is well documented, as is the preference for tumour cells to maintain this state in the presence of $\mathrm{O}_{2}$ (the Warburg effect). To examine the potential therapeutic implications of this, the authors compared two human tumour cell lines - one glycolytic (WiDr, from an adenocarcinoma) and one oxidative ( $\mathrm{SiHa}$, from a cervical squamous carcinoma). In vitro experiments indicated that $\mathrm{SiHa}$ cells preferentially use exogenous lactate, even in the presence of glucose (the normal metabolite for cells undergoing oxidative respiration). However, WiDr cells, which produce lactate as a result of glycolysis, were unaffected by the addition of exogenous lactate, but were unable to survive in the absence of glucose.

Lactate can be exported from or imported into cells through monocarboxylate transporters (MCTs). In oxidative muscle cells MCT1 is expressed at the plasma membrane and mediates the uptake of lactate during supramaximal exercise, whereas glycolytic muscle cells express MCT4 at the plasma membrane, which enables export of lactate. Interestingly, SiHa cells preferentially express MCT1, whereas WiDr cells cultured in vitro preferentially express MCT4. Does the expression of MCT1 explain the capacity of $\mathrm{SiHa}$ cells to make use of exogenous lactate? Inhibition of MCT1 function in these cells prevented lactate uptake and switched the cells to a glycolytic phenotype. Importantly, inhibition of MCT1 in the absence of glucose resulted in the death of SiHa cells, so MCT1 seems to be the main mediator of the ability

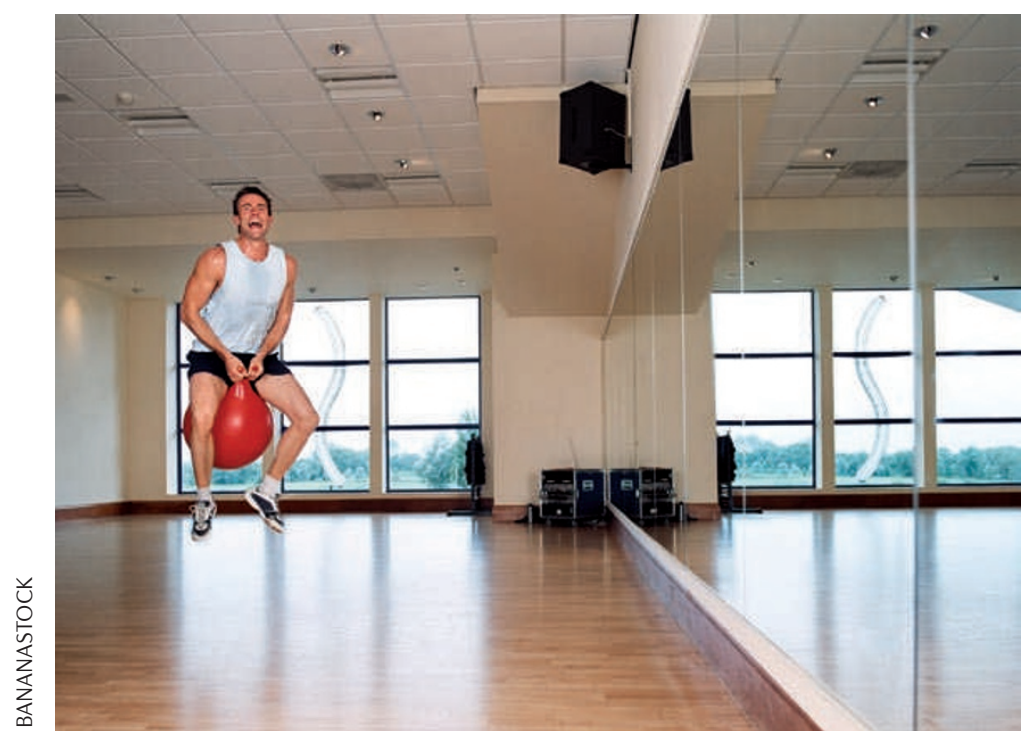

of SiHa cells to import lactate for use in oxidative respiration.

The staining of biopsies from $\mathrm{SiHa}$ and WiDr xenografts indicated that in vivo oxygenated tumour cells express MCT1, whereas those in the hypoxic areas do not. Moreover, treatment of mice with an inhibitor of MCT1 showed that tumours expressing MCT1 had reduced growth whereas MCT1-negative tumours did not. On the basis of these findings the authors hypothesize that oxidative tumour cells close to capillaries use the lactate that is released by the hypoxic tumour cells. The glucose spared by the oxidative cells is able to diffuse into the hypoxic areas of the tumour where it is taken up and used for glycolysis. Therefore, preventing the symbiosis between glycolytic and oxidative cells might be an important therapeutic strategy, particularly as MCT1 is expressed in a wide array of primary human tumour samples.

A further therapeutic implication of the authors' work is the potential to increase tumour sensitivity to radiotherapy. Hypoxia is known to mediate resistance to radiotherapy but, because inhibition of MCT1 switches oxidative cells to glycolysis, $\mathrm{O}_{2}$ consumption decreases and the tumour is reoxygenated. The authors found that treatment of Lewis lung carcinomas in mice with both $6 \mathrm{~Gy}$ of radiation and an MCT1 inhibitor retarded tumour growth significantly more than either treatment alone. 